\title{
Advances in Multi-agent Systems Research: EUMAS 2020 Extended Selected Papers
}

\author{
Nick Bassiliades ${ }^{1} \cdot$ Georgios Chalkiadakis $^{2}$
}

Published online: 22 June 2021

(c) The Author(s), under exclusive licence to Springer Nature Singapore Pte Ltd 2021

This topical collection consists of extended selected papers of the 17th European Conference on Multi-Agent Systems (EUMAS 2020). The conference was originally planned to be held in Thessaloniki, Greece, in April 2020, but due to the COVID19 pandemic, it was eventually held online between September 14-15, 2020. EUMAS 2020 followed the tradition of previous editions (Oxford 2003, Barcelona 2004, Brussels 2005, Lisbon 2006, Hammamet 2007, Bath 2008, Agia Napa 2009, Paris 2010, Maastricht 2011, Dublin 2012, Toulouse 2013, Prague 2014, Athens 2015, Valencia 2016, Evry 2017, Bergen 2018) in aiming to provide the prime European forum for presenting and discussing agents research as the annual designated event of the European Association of Multi-Agent Systems (EURAMAS).

After a rigorous peer-review process, a total of 35 papers were accepted and presented at the conference, covering a wide range of topics in the field of multi-agent systems. Following the conference, in which we had the honor to serve as program co-Chairs, we invited the authors of 8 of those papers to submit extended versions of their work to this SN $\mathrm{CS}$ issue. The authors of 7 of those papers responded to our invitation. The extended papers submitted were then carefully reviewed by at least three reviewers each; and after two rounds of review for most of them, the six (6) papers listed below were selected to be published in this SN CS topical issue. As such, we are confident these papers report on mature research of high technical quality, and do in fact present recent advances in several sub-areas of research in multi-agent systems.

Nick Bassiliades

nbassili@csd.auth.gr

$\triangle$ Georgios Chalkiadakis

gehalk@intelligence.tuc.gr

1 School of Informatics, Aristotle University of Thessaloniki, Thessaloníki, Greece

2 School of Electrical and Computer Engineering, Technical University of Crete, Chania, Greece
As mentioned above, the topical collection consists of six papers. The first paper, by Luca Capezutto, Danesh Tarapore, and Sarvapali D. Ramchurn, puts forward ClusterBased Task Scheduling (CTS), a new algorithm (along with a parallel variant) to solve the Coalition Formation with Spatial and Temporal constraints Problem (CFSTP). CFSTP is a multi-agent task allocation problem where the tasks are spatially distributed, with deadlines and workloads, and in which the number of agents is typically much smaller than that of tasks. Their approach combats complexity and scalability issues plaguing current state-of-the-art algorithms, and improves on their effectiveness when operating in realworld environments where tasks can appear at any time.

The second paper, by Tiago Mota, Mohan Sridharan and Aleš Leonardis, deals with integrated commonsense reasoning and deep learning for transparent decision making in robotics. It presents an architecture that combines the complementary strengths of non-monotonic logical reasoning with incomplete commonsense domain knowledge, deep learning, and inductive learning, enabling a robot to provide on-demand explanations of its decisions, the evolution of associated beliefs, and the outcomes of hypothetical actions, in the form of relational descriptions of relevant domain objects, attributes, and actions.

The third paper, by Farzaneh Farhadi and Nicholas $R$. Jennings, develops the first distributed negotiation mechanism that enables self-interested agents to reach a socially desirable agreement with limited information leakage. The paper puts forward a reinforcement learning-based approach to train agents to learn their optimal strategies in the proposed mechanism; and a heuristic algorithm designed to find close-to-optimal negotiation strategies with reduced computational costs. The proposed mechanism is shown to be budget balanced, and motivates the agents to participate and follow the rules faithfully; while the experimental results confirm that the proposed mechanism significantly outperforms the current state-of-the-art, in terms of social-welfare and privacy leakage. 
The fourth paper, by Emmanouil Rigas and Konstantinos Tsompanidis, presents algorithms to manage congestion in large-scale mobility-on-demand schemes that use electric vehicles that take as input the trip requests and calculate the EV-to-customer assignment, considering the number of executed trips and the utility obtained by the agents. The paper employs Mixed-Integer-Programming (MIP); and also online greedy algorithms with heuristic functions, enhancing their performance with agent-based negotiation.

The fifth paper, by Nikolaos Spanoudakis, is a survey on engineering multi-agent systems with statecharts. The article's purpose is to map the works of the last 20 years in the Agent Oriented Software Engineering and Engineering Multi-Agent Systems fields that use the Statecharts paradigm to aid the software development process, focusing both on the relevant theoretical methods that employ statecharts, but also on identifying the application domains suitable for this kind of modeling.

The sixth paper, by Ronald Chenu Abente Acosta, Nardin Osman, Shen Qiang, Carles Sierra, and Fausto Giunchiglia, deals with the problem of ensuring that an individual's requirements and privacy are respected when they join an online open community, while also respecting the community's ethical code. To this end, the paper builds on the notions of profiles and norms, and designs: (i) a conceptual framework to describe individual and community profiles, including data and norms that provide information about their owner and their requirements; and (ii) a decentralized architecture enabling interactions that leverage the exchange of profile information among people and communities, to ensure that requirements are fulfilled and privacy is respected.

The editors would like to thank all EUMAS authors that accepted our invitation and submitted extended papers to this topical collection, and the reviewers who provided thorough reviews of the submitted papers: indeed, their hard work was what allowed us to put together a strong collection of papers. We would also like to thank the SN Computer Science Journal and especially Mrs. Teena Bedi for facilitating the publication of this topical collection with selected EUMAS 2020 extended papers.

Nick Bassiliades

Georgios Chalkiadakis

Publisher's Note Springer Nature remains neutral with regard to jurisdictional claims in published maps and institutional affiliations. 